\title{
Assessment of Olfactory Responses of Bactrocera zonata, B. cucurbitae and B. Dorsalis (Diptera: Tephritidae) to different biolure baits and Combined Use of Male Annihilation Technique (MAT) and sterile insect technique (SIT) in IPM programme for Bactorecera Fruit Flies
}

\author{
Muhammad Nabeel Saleem* \\ Department of Entomology, University of Agriculture Faisalabad, Pakistan \\ *Corresponding Author: Muhammad Nabeel Saleem, Department of Entomology, University of Agriculture Faisalabad, Punjab, \\ Pakistan.
}

Received: May 20, 2019; Published: June 19, 2019

DOI: 10.31080/ASAG.2019.03.0535

Present study was carried out to evaluate olfactory response of B. zonata, B. dorsalis and B. eucurabitae towards different BiolureBaits. The results of ecperiment-1 revealed that Mixture-V (Beef and Fish extracts, Yeast, Starch, rose oil, Protein-hydrolysate, vinegar, casein and cidar-oil mixed in a raio of 0.125 with 1 part of banana squash) attracted maximum B. zonata (27\% males and $29 \%$ females flies), B. dorsalis (27.4\% males and $28.7 \%$ females) and B. cucurbitae (27\% male and $29.72 \%$ female flies) followed by mixture-IV (beef and fish extracts, yeast, starch , rose oil, proteinhydrolysate, vinegar, casein and cidar-oil mixed in ratio of 0.25 with 1 part of banana squash) which attracted significantly higher density of B. zonata (24.4\% males and $28.6 \%$ females), B. dorsalis ( 25\% male and $29.65 \%$ females) and B. cucurbitae (25.65\% male and $27.25 \%$ female) as compared to GF -120 (23\% male and $29 \%$ female). The results of experiment- 2 demonstrated that admixture-IV (0.25 parts of highly attractive mixture of experiment-1 mixed with 0.25 parts of each of ammonium acetate, DAP, acetic acid with auxiliary) attracted maximum percentage of B. zonata (35.71\% male and $36.72 \%$ female), B. dorsalis (35\% male and $36.73 \%$ female) and B. cucurbitae (36\% male and \#\&.7\% female) followed by admixture-V $(0.125$ parts of highly attractive mixture of experiment-1 mixed with 0.125 parts of each of ammonium acetate, DAP, acetic acid with auxiliary) which attracted significantly higher density of B. zonata (32.26\% males and $33.255 \%$ female), B. dorsalis (32.7\% male and $33.7 \%$ female) and B. cucurbitae ( $33.4 \%$ male and $35 \%$ female) as compared to GF-120 (12\% male and $13 \%$ female). The results of experiment 3 exhibited that all the biolure proved significantly more attractive to three fruit fly species when biopesticides were admixed at lower concentration (1 ppm). Biolure- Bait- I ( admixture-IV baited with 1ppm of metarhiziium anisopliae) attracted higher percentage of released
B. zonata (10.1 - 16.59\%), B. dorsalis (11.25 - 5.5\% starved) and B. cucurbitae ( 11.58 - 16.35\%) followed by biolure - bait-II (admixture-IV baited with $1 \mathrm{ppm}$ of beauveria bassiana) which attracted statistically reasonable percentage of B. zonata (10.1-15.75\%), $B$. dorsalis (10.24-14.82\%) and B. cucurbitae (10.75-14.58\%). The order of the effectiveness of the finally developed biolure-baits was biolure-bait-I > biolure-bait-VI > biolure-baits-II > biolure -bait-III $>$ biolure-bait-IV $>$ biolure-bait-V $>$ biolure-bait-V $>$ Biolure-bait-XI $>$ Biolure-bait-VI $>$ Biolure-bait-VII $>$ Biolure-bait-VIII $>$ Biolurebait-IX > Biolure-bait-X.

Fruit flies are amongst the most damaging insect pests causing more than USD 200 million losses to horticultural crops in Pakistan and impending the horticultural exports, the use of synthetic insecticides remained insufficient to manage fruit flies populations and the presence of their residues is another reason for restricting the horticultural exports. The application of male annihilation technique (MAT) incorporating the methyl eugenol (ME) (1,2-dimethoxy - 4 -(2-propenyl)benzene), a phenyl propaniod compound occurring in many plant species, is being applied as a component of area-wide integrated pest management (AW-IPM) programmes. The MAT is an environmentally-friendly, lure-and-kill the males approach. The sterile insect technique (SIT) which is likewise an environmentally friendly technique is also being applied for management of fruit flies. Both techniques can be integrated in AWIPM programmes. The integration of both techniques has so far been sequential with the SIT applied after a significant reduction of the wild population with the MAT to avoid killing too many of the released sterile males in ME-baited traps/devices, which would significantly reduce the efficacy of the SIT. There is, however, potential for simultaneously implementing the SIT and the MAT as 
Bactrocera dorsalis males once fed on ME showed significantly less tendency for repeat feeding on ME. Additionally, Feeding on ME is known to also enhance the mating competitiveness of certain Bactrocera spp. Male which can increase the effectiveness manifold of management program incorporating the SIT. Currently used systems for holding the mass-reared males in fly release facilities does not allow for application of ME through feeding. Therefore, delivery system of ME that would be applicable in fly emergence and release facilities was developed. The development for devising the system for application of ME in a mass-rearing facility and its implication for effectiveness of SIT are discussed.

Volume 3 Issue 7 July 2019

(c) All rights are reserved by Muhammad Nabeel Saleem.

Citation: Muhammad Nabeel Saleem. "Assessment of Olfactory Responses of Bactrocera zonata, B. cucurbitae and B. Dorsalis (Diptera: Tephritidae) to different biolure baits and Combined Use of Male Annihilation Technique (MAT) and sterile insect technique (SIT) in IPM programme for Bactorecera Fruit Flies". Acta Scientific Agriculture 3.7 (2019): 155-156. 